\title{
Psychosocial effects of radiotherapy after mastectomy
}

\author{
A V M HUGHSON, A F COOPER, C S McARDLE, D C SMITH
}

\begin{abstract}
Psychosocial morbidity was measured in 47 patients who received postoperative radiotherapy and in 38 who received no further treatment after mastectomy. Roughly one third of all patients experienced depression or anxiety. One month after operation, before radiotherapy, there were no significant differences between the two groups in any of the measures of psychosocial morbidity. Knowledge of impending treatment did not seem to influence morbidity. At three months patients who had completed radiotherapy had significantly more somatic symptoms and social dysfunction than those not so treated. At six months the radiotherapy group continued to show more somatic symptoms, but a year after operation there were no significant differences between the groups.

Although several patients who received radiotherapy were upset by their treatment, the study failed to confirm that depression and anxiety were commoner among those given radiotherapy than among patients given no further treatment.
\end{abstract}

\section{Introduction}

There is general agreement that radiotherapy after mastectomy reduces the rate of local recurrence but does not influence systemic relapse or survival. ${ }^{\prime}$ Nevertheless, a recent survey showed that postoperative radiotherapy is still widely used in the United Kingdom. ${ }^{2}$ Psychological morbidity is common after mastectomy ${ }^{3}$ and is increased after adjuvant chemotherapy. ${ }^{45}$ Previous studies suggested that it may also be increased by postoperative radiotherapy, but the extent of the morbidity is uncertain because of lack of enough control subjects. ${ }^{6-11}$ The present study sought to show

University Department of Psychological Medicine, Glasgow G12 0AA

A V M HUGHSON, MB, MRCPSYCH, honorary clinical lecturer

Divisions of Psychiatry and Surgery, Victoria Infirmary, Glasgow

A F COOPER, MD, FRCPSYCH, consultant psychiatrist

D C SMITH, MB, FRCS, consultant surgeon

Department of Surgery, Royal Infirmary, Glasgow

C S MCARDLE, MD, FRCS, consultant surgeon

Correspondence to: Dr Mark Hughson, 1 Cleveden Gardens, Glasgow G12 OPU. whether postoperative radiotherapy induced more psychological and social morbidity than that seen in a control group who had mastectomy but no further treatment.

\section{Patients and methods}

Consecutive patients under the age of 70 with stage I or II breast cancer were included in the study. All patients underwent simple mastectomy and axillary clearance. Patients with histologically proved stage II cancer started radiotherapy four to six weeks after mastectomy; those without nodal spread received no further local treatment. Postoperative orthovoltage radiotherapy ( 15 fractions over three weeks) was administered to the chest wall, axilla, infraclavicular and supraclavicular fossas, and internal mammary region (average tumour dose $37 \cdot 8 \mathrm{~Gy}$ ).

Psychological morbidity was measured with observer rating scales developed by Maguire to study a population after mastectomy. ${ }^{11}$ The scales covered various aspects of psychosocial morbidity: depression, anxiety, loss of libido, lethargy, social dysfunction, and inability to work. For each scale four point ratings were used ( $0=$ absent, $1=$ mild, $2=$ moderate, $3=$ severe). Self rating scales were also used-namely, the Leeds general scales for self assessment of depression and anxiety ${ }^{12}$ and the general health questionnaire. ${ }^{13}$ The Leeds scales were modified slightly to cover the same time period as the general health questionnaire and the observer scales. Thus all scales measured morbidity experienced over the few weeks before each assessment. As the general health questionnaire contained somatic and social items that might have been endorsed by patients with purely physical distress the four subscales (somatic symptoms, anxiety and insomnia, social dysfunction, and severe depression) were analysed separately. Scores on each Leeds scale ranged from 0 to 18 . On the general health questionnaire scores ranged from 0 to 60 , while for each subscale of the general health questionnaire scores ranged from 0 to 21 .

Psychological assessments were performed one month after operation (before patients with stage II disease had started radiotherapy) and again three months after operation (two to three weeks after completion of radiotherapy). Patients also completed the Eysenck personality inventory (form A) at the one month interview ${ }^{14}$ and the Mill Hill synonyms test of verbal intelligence at three months. ${ }^{15} 16$ Patients who had completed radiotherapy were then randomised to receive chemotherapy or no further treatment within the framework of a pre-existing clinical trial. ${ }^{17}$ In patients who had received radiotherapy alone two further assessments were made, at six and 13 months after operation.

A simple arbitrary score for physical symptoms was devised based on the presence or absence of several key symptoms in the few weeks before each assessment. These included anorexia, nausea, vomiting, sore throat, dysphagia, skin reaction, pain, and arm swelling. One point was allotted for each symptom present.

All but 15 of the 288 interviews in the study were conducted by AVMH, mostly in the patients' homes. Sixteen patients were assessed jointly by 
AVMH and AFC to check reliability. At the assessment at six months the 23 patients who had received radiotherapy alone were asked, "How do you feel about your treatment?" Their spontaneous replies were recorded verbatim.

Data analysis-Cohen's kappa was used to assess inter-rater reliability for the Maguire scales. ${ }^{18}$ Demographic and other features of the two treatment groups were compared with $\chi^{2}$ or two tailed $t$ tests as appropriate. Results obtained with the observer rating scales were analysed by standard error tests comparing the proportions of patients experiencing some and no morbidity, with correction for continuity as appropriate.$^{19}$ Results of the self rating scales were analysed by the Mann-Whitney U test. As previous studies suggested that patients allocated to radiotherapy would experience more psychological, social, and physical morbidity than those allocated no further treatment, and to give the maximum chance of detecting differences, one tailed tests of significance were used to compare scores at each of the four assessments.

\section{Results}

Forty three patients with stage I disease and 51 with stage II disease were asked to participate in the study, but four in each group refused to do so.

TABLE I-Patient characteristics

\begin{tabular}{|c|c|c|c|}
\hline & \multicolumn{2}{|c|}{ Treatment after mastectomy } & \multirow[b]{2}{*}{ p Value } \\
\hline & None $(n=39)$ & Radiotherapy $(n=47)$ & \\
\hline Mean (SD) age at operation (years) & $53.9(9 \cdot 5)$ & $51 \cdot 1(11 \cdot 5)$ & NS \\
\hline $\begin{array}{l}\text { No (\%) in social class: } \\
\text { I + II } \\
\text { III } \\
\text { IV + V }\end{array}$ & $\begin{array}{r}15(38 \cdot 4) \\
20(51 \cdot 3) \\
4(10 \cdot 3)\end{array}$ & $\begin{array}{r}18(38 \cdot 3) \\
23(48 \cdot 9) \\
6(12 \cdot 8)\end{array}$ & NS \\
\hline $\begin{array}{l}\text { Marital state }(\mathrm{No}(\%)): \\
\text { Married } \\
\text { Divorced } \\
\text { Widowed } \\
\text { Never married }\end{array}$ & $\begin{array}{l}32(82 \cdot 0) \\
1(2 \cdot 6) \\
5(12 \cdot 8) \\
1(2 \cdot 6)\end{array}$ & $\begin{array}{l}30(63 \cdot 8) \\
4(8 \cdot 5) \\
7(14 \cdot 9) \\
6(12 \cdot 8)\end{array}$ & NS $^{\star}$ \\
\hline $\begin{array}{l}\text { No }(\%) \text { having had previous psychiatric } \\
\text { No treatment } \\
\text { From general practitioner } \\
\text { From psychiatrist }\end{array}$ & $\begin{array}{l}\text { treatment: } \\
\qquad \begin{array}{l}28(71 \cdot 8) \\
10(25 \cdot 6) \\
1(2 \cdot 6)\end{array}\end{array}$ & $\begin{array}{c}30(63 \cdot 8) \\
15(31 \cdot 9) \\
2(4 \cdot 3)\end{array}$ & NSt \\
\hline $\begin{array}{l}\text { Mean (SD) score on Eysenck personalit } \\
\text { Extraversion } \\
\text { Neuroticism } \\
\text { Mean (SD) verbal intelligence quotient }\end{array}$ & $\begin{array}{l}\text { inventory: } \\
11 \cdot 5(3 \cdot 8) \\
9 \cdot 9(5 \cdot 0) \\
105 \cdot 3(11 \cdot 5)\end{array}$ & $\begin{array}{c}10 \cdot 5(3 \cdot 3) \\
8 \cdot 5(5 \cdot 4) \\
101 \cdot 2(11 \cdot 2)\end{array}$ & $\begin{array}{l}\text { NS } \\
\text { NS } \\
\text { NS }\end{array}$ \\
\hline
\end{tabular}

$\chi^{2}$ Test on married $v$ unmarried. $\nmid \chi^{2}$ Test on some $v$ none.

TABLE II-Comparison between treatment groups of scores above zero on observer ratings at one and three months $(0=a b s e n t, 1=$ mild, $2=$ moderate, $3=$ severe $)$

\begin{tabular}{|c|c|c|c|c|c|c|c|c|c|c|c|c|}
\hline & \multicolumn{12}{|c|}{ Months after mastectomy } \\
\hline & \multicolumn{6}{|c|}{ One } & \multicolumn{6}{|c|}{ Three } \\
\hline & \multicolumn{4}{|c|}{ Score } & \multirow{2}{*}{\multicolumn{2}{|c|}{ No*$^{\star}$ p Valuef }} & \multicolumn{4}{|c|}{ Score } & \multirow{2}{*}{\multicolumn{2}{|c|}{ No` $p$ Value }} \\
\hline & 1 & 2 & 3 & $(\%>0)$ & & & 1 & 2 & 3 & $(\%>0)$ & & \\
\hline $\begin{array}{l}\text { Depression: } \\
\text { No treatment } \\
\text { Radiotherapy }\end{array}$ & $\begin{array}{l}12 \\
14\end{array}$ & $\begin{array}{l}2 \\
3\end{array}$ & 1 & $\begin{array}{l}(37) \\
(38)\end{array}$ & $\left.\begin{array}{l}38 \\
47\end{array}\right\}$ & NS & $\begin{array}{r}7 \\
12\end{array}$ & $\begin{array}{l}2 \\
2\end{array}$ & & $\begin{array}{l}(24) \\
(30)\end{array}$ & $\left.\begin{array}{l}38 \\
46\end{array}\right\}$ & NS \\
\hline $\begin{array}{l}\text { Anxiety: } \\
\text { No treatment } \\
\text { Radiotherapy }\end{array}$ & $\begin{array}{r}13 \\
8\end{array}$ & $\begin{array}{l}2 \\
5\end{array}$ & 3 & $\begin{array}{l}(39) \\
(34)\end{array}$ & $\left.\begin{array}{l}38 \\
47\end{array}\right\}$ & NS & $\begin{array}{l}7 \\
7\end{array}$ & $\begin{array}{l}4 \\
4\end{array}$ & & $\begin{array}{l}(29) \\
(24)\end{array}$ & $\left.\begin{array}{l}38 \\
46\end{array}\right\}$ & NS \\
\hline $\begin{array}{r}\text { Loss of libido } \neq: \\
\text { No treatment } \\
\text { Radiotherapy }\end{array}$ & $\begin{array}{l}4 \\
3\end{array}$ & $\begin{array}{l}5 \\
4\end{array}$ & $\begin{array}{l}5 \\
9\end{array}$ & $\begin{array}{l}(45) \\
(52)\end{array}$ & $\left.\begin{array}{l}31 \\
31\end{array}\right\}$ & NS & $\begin{array}{l}5 \\
2\end{array}$ & $\begin{array}{l}3 \\
6\end{array}$ & $\begin{array}{l}3 \\
7\end{array}$ & $\begin{array}{l}(34) \\
(48)\end{array}$ & $\left.\begin{array}{l}32 \\
31\end{array}\right\}$ & NS \\
\hline $\begin{array}{l}\text { Lethargy: } \\
\text { No treatment } \\
\text { Radiotherapy }\end{array}$ & $\begin{array}{l}12 \\
21\end{array}$ & $\begin{array}{l}7 \\
6\end{array}$ & 2 & $\begin{array}{l}(50) \\
(62)\end{array}$ & $\left.\begin{array}{l}38 \\
47\end{array}\right\}$ & NS & $\begin{array}{r}8 \\
18\end{array}$ & $\begin{array}{c}3 \\
12\end{array}$ & & $\begin{array}{l}(29) \\
(65)\end{array}$ & $\left.\begin{array}{l}38 \\
46\end{array}\right\}$ & $<0.001$ \\
\hline $\begin{array}{l}\text { Social dysfunctio } \\
\text { No treatment } \\
\text { Radiotherapy }\end{array}$ & $\begin{array}{l}\mathrm{n}: \\
12 \\
13\end{array}$ & $\begin{array}{l}2 \\
4\end{array}$ & 1 & $\begin{array}{l}(37) \\
(38)\end{array}$ & $\left.\begin{array}{l}38 \\
47\end{array}\right\}$ & NS & $\begin{array}{r}6 \\
12\end{array}$ & $\begin{array}{l}2 \\
6\end{array}$ & & $\begin{array}{l}(21) \\
(39)\end{array}$ & $\left.\begin{array}{l}38 \\
46\end{array}\right\}$ & $<0.04$ \\
\hline $\begin{array}{l}\text { Inability to work } \\
\text { No treatment } \\
\text { Radiotherapy }\end{array}$ & $\begin{array}{l}14 \\
17\end{array}$ & $\begin{array}{l}9 \\
9\end{array}$ & & $\begin{array}{l}(61) \\
(55)\end{array}$ & $\left.\begin{array}{l}38 \\
47\end{array}\right\}$ & NS & $\begin{array}{l}10 \\
11\end{array}$ & $\begin{array}{l}1 \\
6\end{array}$ & & $\begin{array}{l}(29) \\
(37)\end{array}$ & $\left.\begin{array}{l}38 \\
46\end{array}\right\}$ & NS \\
\hline
\end{tabular}

*One patient with stage I disease could not attend the one month interview and another the three month; one patient with stage II disease refused the three month interview. three month; one patient with stage II disease refused the three month

†One tailed test comparing proportions
$\ddagger$ Married/cohabiting patients only.
TABLE III-Comparison between treatment groups of scores on self rating scales at one and three months

\begin{tabular}{|c|c|c|c|c|c|}
\hline & \multicolumn{5}{|c|}{ Months after mastectomy } \\
\hline & \multicolumn{2}{|c|}{ One } & \multicolumn{3}{|c|}{ Three } \\
\hline & $\begin{array}{l}\text { Median (inter- } \\
\text { quartile range) }\end{array}$ & $\mathrm{No}^{\star} \mathrm{p}$ Value & $\begin{array}{l}\text { Median (inter- } \\
\text { quartile range) }\end{array}$ & $\mathrm{No}^{\star}$ & p Valuet \\
\hline \multicolumn{6}{|c|}{ General health questionnaire } \\
\hline $\begin{array}{l}\text { No treatment } \\
\text { Radiotherapy }\end{array}$ & $\begin{array}{l}4 \cdot 0(1-12) \\
5 \cdot 0(2-15)\end{array}$ & $\left.\begin{array}{l}38 \\
47\end{array}\right\} \mathrm{NS}$ & $\begin{array}{l}2 \cdot 0(0-8) \\
4 \cdot 0(1-16)\end{array}$ & $\left.\begin{array}{l}38 \\
46\end{array}\right\}$ & $<0.04$ \\
\hline $\begin{array}{l}\text { Somatic symptom } \\
\text { No treatment } \\
\text { Radiotherapy }\end{array}$ & $\begin{array}{l}3 \cdot 0(1-5) \\
3 \cdot 0(2-6)\end{array}$ & $\left.\begin{array}{l}38 \\
47\end{array}\right\} \mathrm{NS}$ & $\begin{array}{l}2 \cdot 0(1-5) \\
4 \cdot 0(2-7)\end{array}$ & $\left.\begin{array}{l}38 \\
46\end{array}\right\}$ & $<0.05$ \\
\hline $\begin{array}{l}\text { Anxiety and inson } \\
\text { No treatment } \\
\text { Radiotherapy }\end{array}$ & ia: $\begin{array}{r}3.0(0-7) \\
3.0(1-7)\end{array}$ & $\left.\begin{array}{l}38 \\
47\end{array}\right\}$ NS & $\begin{array}{l}4 \cdot 5(2-7) \\
3 \cdot 0(1-7)\end{array}$ & $\left.\begin{array}{l}38 \\
46\end{array}\right\}$ & NS \\
\hline $\begin{array}{l}\text { Social dysfunctior } \\
\text { No treatment } \\
\text { Radiotherapy }\end{array}$ & $\begin{array}{l}7 \cdot 5(7-9) \\
8 \cdot 0(7-10)\end{array}$ & $\left.\begin{array}{l}38 \\
47\end{array}\right\}$ NS & $\begin{array}{l}7 \cdot 0(7-8) \\
7 \cdot 0(7-10)\end{array}$ & $\left.\begin{array}{l}38 \\
46\end{array}\right\}$ & $<0.07$ \\
\hline $\begin{array}{l}\text { Severe depression } \\
\text { No treatment } \\
\text { Radiotherapy }\end{array}$ & $\begin{array}{l}0.0(0-1) \\
0.0(0-1)\end{array}$ & $\left.\begin{array}{l}38 \\
47\end{array}\right\} \mathrm{NS}$ & $\begin{array}{l}0.0(0-2) \\
0.0(0-1)\end{array}$ & $\left.\begin{array}{l}38 \\
46\end{array}\right\}$ & NS \\
\hline \multicolumn{6}{|c|}{ Leeds general scales } \\
\hline $\begin{array}{l}\text { Depression: } \\
\text { No treatment } \\
\text { Radiotherapy }\end{array}$ & $\begin{array}{l}1 \cdot 0(0-3) \\
2 \cdot 0(1-5)\end{array}$ & $\left.\begin{array}{l}38 \\
47\end{array}\right\} \mathrm{NS}$ & $\begin{array}{l}2 \cdot 0(0-4) \\
2 \cdot 0(0-5)\end{array}$ & $\left.\begin{array}{l}38 \\
46\end{array}\right\}$ & NS \\
\hline $\begin{array}{l}\text { Anxiety: } \\
\text { No treatment } \\
\text { Radiotherapy }\end{array}$ & $\begin{array}{l}4.0(1-7) \\
3.0(0 \cdot 7)\end{array}$ & $\left.\begin{array}{l}38 \\
47\end{array}\right\} \mathrm{NS}$ & $\begin{array}{l}3.0(1-7) \\
2.0(0.6)\end{array}$ & $\left.\begin{array}{l}38 \\
46\end{array}\right\}$ & NS \\
\hline
\end{tabular}

$\star$ See Table II.

tOne tailed probability, Mann-Whitney U test.

TABLE IV-Comparison between treatment groups of scores above zero on observer ratings at six and 13 months

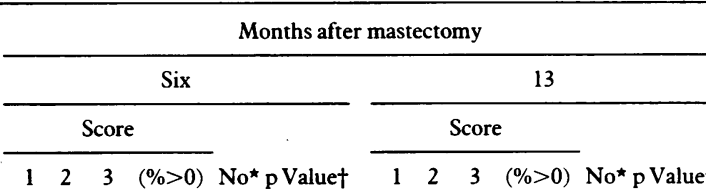

Depression:

$\left.\begin{array}{llllll}\text { No treatment } & 5 & 1 & & (16) & 38 \\ \text { Radiotherapy } & 1 & 1 & 1 & (13) & 23\end{array}\right\}$ NS $\left.\quad \begin{array}{llllll}6 & & 1 & (19) & 37 \\ 2 & 1 & & (14) & 21\end{array}\right\}$ NS

Anxiety:

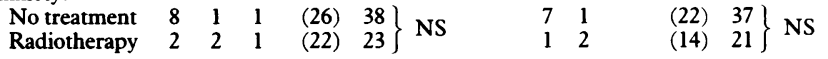

Loss of libido $\ddagger$ :

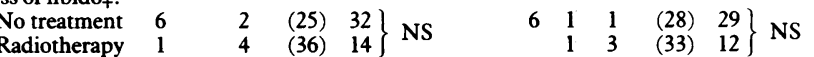

Lethargy:

$\left.\begin{array}{llllrl}\text { No treatment } & 6 & & (16) & 38 \\ \text { Radiotherapy } & 4 & 3 & (30) & 23\end{array}\right\}$ NS $\left.\quad \begin{array}{rllll}12 & & & (32) & 37 \\ 5 & 2 & 2 & (43) & 21\end{array}\right\}$ NS

Social dysfunction:

Social dysfunction:

$\begin{array}{ll}\text { No treatment } & 5 \\ \text { Radiotherapy } & 1\end{array}$

Inability to work:

$\begin{array}{lll}\text { No treatment } & 3 & \\ \text { Radiotherapy } & 2 & 2\end{array}$

$\left.\begin{array}{ll}\text { (13) } & 38 \\ \text { (13) } & 23\end{array}\right\}$ NS

$\left.\begin{array}{llll}4 & 1 & (14) & 37 \\ 1 & 2 & (14) & 21\end{array}\right\}$ NS

(8) $\begin{aligned} & 38 \\ & \text { (17) }\end{aligned} 23$ NS

$\left.\begin{array}{lllll}2 & 2 & (11) & 37 \\ 2 & 2 & 1 & (24) & 21\end{array}\right\}$ NS

^Patients randomised to chemotherapy after three months were excluded; one patient with stage I disease refused the 13 month interview, and two patients with stage II disease died after six months.

tOne tailed test comparing proportions with some and no morbidity.

$¥$ Married/cohabiting patients only.

Table I shows that the demographic and other background data for the two groups were similar. For the Maguire scales the values of Cohen's kappa for depression, anxiety, lethargy, loss of libido, social dysfunction, and inability to work were respectively $0 \cdot 74,0 \cdot 68,0 \cdot 81,1 \cdot 00,0 \cdot 85$, and $0 \cdot 90$. Thus interrater reliability was satisfactory.

Tables II and III show the scores on the observer and self rating scales at one and three months after operation for the two groups. At one month, before treatment, there were only minor differences between the groups, none of which were significant. At three months patients who had completed radiotherapy two to three weeks previously showed significantly higher scores for lethargy $(p<0.001)$ and social dysfunction $(p<0.04)$ on the observer rating scales than those not so treated. They also scored significantly higher on the general health questionnaire $(\mathbf{p}<0.04)$ and on its somatic 
symptoms subscale $(\mathrm{p}<0.05)$. They tended to score higher on the social dysfunction subscale $(\mathrm{p}<0.07)$.

At six months observer ratings showed that the trend towards excess lethargy in patients who had received radiotherapy was maintained (table IV). These patients scored higher on the general health questionnaire $(p<0.04)$ and the somatic symptom subscale $(p<0.04)$ (table V). Neither observer nor self ratings showed any excess of anxiety or depression in this group. By 13 months the trend towards excess lethargy and somatic symptoms in the patients treated with radiotherapy had stopped. Although there were no significant differences between the groups in any of the scales, there was a slight trend towards more anxiety and depression in the patients not given radiotherapy.

Scores for physical symptoms (table VI) were similar at one month after operation but at three months were considerably higher in the group given radiotherapy $(p<0.0001)$. Thereafter there were no significant differences between the groups.

TABLE V-Comparison between treatment groups of scores on self rating scales at six and 13 months

\begin{tabular}{|c|c|c|c|c|c|}
\hline & \multicolumn{5}{|c|}{ Months after mastectomy } \\
\hline & \multicolumn{2}{|c|}{ Six } & \multicolumn{3}{|c|}{13} \\
\hline & $\begin{array}{l}\text { Median (inter- } \\
\text { quartile range) }\end{array}$ & $\mathrm{No}^{\star} \mathrm{p}$ Valuef & $\begin{array}{l}\text { Median (inter- } \\
\text { quartile range) }\end{array}$ & $\mathrm{No}^{\star}$ & p Valuet \\
\hline \multicolumn{6}{|c|}{ General health questionnaire } \\
\hline $\begin{array}{l}\text { No treatment } \\
\text { Radiotherapy }\end{array}$ & $\begin{array}{l}0.0(0-4) \\
2 \cdot 0(0-12)\end{array}$ & $\left.\begin{array}{l}38 \\
23\end{array}\right\}<0.04$ & $\begin{array}{l}1 \cdot 0(0-12) \\
0.0(0-3)\end{array}$ & $\left.\begin{array}{l}37 \\
19\end{array}\right\}$ & NS \\
\hline $\begin{array}{c}\text { Somatic symptom } \\
\text { No treatment } \\
\text { Radiotherapy }\end{array}$ & $\begin{array}{l}2 \cdot 0(1-4) \\
4 \cdot 0(1-8)\end{array}$ & $\left.\begin{array}{l}38 \\
23\end{array}\right\}<0.04$ & $\begin{array}{l}3 \cdot 0(2-6) \\
3 \cdot 0(1-5)\end{array}$ & $\left.\begin{array}{l}37 \\
19\end{array}\right\}$ & NS \\
\hline $\begin{array}{l}\text { Anxiety and inso } \\
\text { No treatment } \\
\text { Radiotherapy }\end{array}$ & ia: $\begin{array}{l}3.0(1-6) \\
3.0(1-7)\end{array}$ & $\left.\begin{array}{l}38 \\
23\end{array}\right\}$ NS & $\begin{array}{l}4 \cdot 0(2-8) \\
3 \cdot 0(1-6)\end{array}$ & $\left.\begin{array}{l}37 \\
19\end{array}\right\}$ & NS \\
\hline $\begin{array}{c}\text { Social dysfunctio } \\
\text { No treatment } \\
\text { Radiotherapy }\end{array}$ & $\begin{array}{l}7 \cdot 0(7-7) \\
7 \cdot 0(7-8)\end{array}$ & $\left.\begin{array}{l}38 \\
23\end{array}\right\} \mathrm{NS}$ & $\begin{array}{l}7 \cdot 0(7-8) \\
7 \cdot 0(7-7)\end{array}$ & $\left.\begin{array}{l}37 \\
19\end{array}\right\}$ & NS \\
\hline $\begin{array}{l}\text { Severe depressior } \\
\text { No treatment } \\
\text { Radiotherapy }\end{array}$ & $\begin{array}{l}0.0(0-1) \\
0.0(0-0)\end{array}$ & $\left.\begin{array}{l}38 \\
23\end{array}\right\} \mathrm{NS}$ & $\begin{array}{l}0.0(0-1) \\
0.0(0-0)\end{array}$ & $\left.\begin{array}{l}37 \\
19\end{array}\right\}$ & NS \\
\hline \multicolumn{6}{|c|}{ Leeds general scales } \\
\hline $\begin{array}{l}\text { Depression: } \\
\text { No treatment } \\
\text { Radiotherapy }\end{array}$ & $\begin{array}{l}1 \cdot 0(0-3) \\
2 \cdot 0(0-3)\end{array}$ & $\left.\begin{array}{l}38 \\
23\end{array}\right\} \mathrm{NS}$ & $\begin{array}{l}3 \cdot 0(1-5) \\
2 \cdot 0(0-4)\end{array}$ & $\left.\begin{array}{l}37 \\
19\end{array}\right\}$ & NS \\
\hline $\begin{array}{l}\text { Anxiety: } \\
\text { No treatment } \\
\text { Radiotherapy }\end{array}$ & $\begin{array}{l}2 \cdot 5(1-6) \\
2 \cdot 0(0-6)\end{array}$ & $\left.\begin{array}{l}38 \\
23\end{array}\right\}$ NS & $\begin{array}{l}3.0(1-7) \\
1.0(0-3)\end{array}$ & $\left.\begin{array}{l}37 \\
19\end{array}\right\}$ & NS \\
\hline
\end{tabular}

* See table IV; also at 13 months two patients felt too ill to complete self rating scales. tOne tailed probability, Mann-Whitney U test.

TABLE VI-Median scores and interquartile ranges $(I Q R)$ for physical symptoms

\begin{tabular}{|c|c|c|c|c|c|c|c|c|}
\hline & \multicolumn{8}{|c|}{ Months after mastectomy } \\
\hline & \multicolumn{2}{|l|}{ One } & \multicolumn{2}{|l|}{ Three } & \multicolumn{2}{|l|}{ Six } & \multicolumn{2}{|l|}{13} \\
\hline & Median (IQR & $\mathrm{No}^{\star}$ & Median (IQR) & $\mathrm{No}^{\star}$ & Median (IQF & $\mathrm{No}^{\star}$ & Median (IQF & $\mathrm{No}^{\star}$ \\
\hline $\begin{array}{l}\text { No treatment } \\
\text { Radiotherapy }\end{array}$ & $\begin{array}{l}0.0(0-1) \\
0.0(0-1)\end{array}$ & $\begin{array}{l}38 \\
47\end{array}$ & $\begin{array}{l}0.0(0-0) \\
3 \cdot 0+(2-4)\end{array}$ & $\begin{array}{l}38 \\
46\end{array}$ & $\begin{array}{l}0.0(0-0) \\
0.0(0-1)\end{array}$ & $\begin{array}{l}38 \\
23\end{array}$ & $\begin{array}{l}0.0(0-0) \\
0.0(0-1)\end{array}$ & $\begin{array}{l}37 \\
21\end{array}$ \\
\hline
\end{tabular}

*See tables II-IV.

tp $<0 \cdot 0001$ (one tailed probability, Mann-Whitney U test).

\section{Discussion}

There have been several studies of psychological morbidity after mastectomy in which at least some patients received postoperative radiotherapy. Morris et al in a series of 69 patients, found that radiotherapy had no effect on psychological morbidity two years after mastectomy but did not report the early effects of treatment. ${ }^{20}$ In a study of 75 patients, of whom 62 received radiotherapy, Maguire et al were unable to quantify the psychological consequences but thought radiotherapy might increase emotional distress. ${ }^{1}$ Other studies, with fewer subjects, also suggested that emotional upset after mastectomy may be increased by radiotherapy. ${ }^{68-10}$

This study showed that one month after mastectomy more than a third of all patients reported depression, anxiety, or social dysfunction and well over half some degree of lethargy or inability to work. There were no significant differences, however, in any of the measures of psychosocial morbidity between those patients who were due to receive radiotherapy and those who were not. Knowledge of impending treatment did not seem to cause excess morbidity.

Two to three weeks after completing treatment (that is, at the three month assessment) almost two thirds of the group given radiotherapy were found on observer ratings to have been lethargic over the previous few weeks compared with under a third of the control group. Nearly half of the patients given radiotherapy had experienced social dysfunction-for example, feeling indecisive or uncommunicative, or failing to enjoy their usual activitiescompared with just over a third of those who did not receive radiotherapy. A quarter or more of all patients were judged to have been depressed or anxious, but there were no significant differences between the two groups. Psychological distress of clinical degree (namely, observer ratings of two or above) was surprisingly uncommon. Results of the self rating scales were in keeping with those of the observer ratings scales. There were also no significant differences in depression or anxiety at six and 13 months. Though the available sample of patients treated with radiotherapy was smaller from six months onwards, it was of sufficient size to show that a significant excess of somatic symptoms persisted at six months.

Might the excess of somatic symptoms seen at three and six months in patients treated with radiotherapy have been a psychological rather than a physical effect of treatment? Though psychological stress might induce somatic symptoms even in the absence of overt depression or anxiety, there seems no reason why patients treated with radiotherapy should be any more likely to show psychological stress as somatic symptoms than patients not so treated. Thus the excess of somatic symptoms probably had a physical basis. For similar reasons the excess of social dysfunction noted at three months in the patients given radiotherapy seems unlikely to have been psychogenic. Patients travelling to hospital five days a week for a treatment that often made them feel tired and caused skin reactions might be expected to report excess physical symptoms and social dysfunction.

As all the patients treated with radiotherapy had stage II cancer, while those not so treated had stage I cancer, results might conceivably reflect severity of disease rather than treatment with radiotherapy. This possibility, however, seems unlikely. Stage II disease might affect patients in two main ways: firstly, the patients might realise that their disease has a relatively poor outlook and, secondly, there is a remote chance that an excess of micrometastases might alter mood physiologically. In either case increased depression or anxiety would seem likely. Yet despite this potential bias no excess of psychological morbidity was found in patients with stage II disease.

The failure to show that radiotherapy induced an excess of depression or anxiety at any time in the first year after operation was unexpected. Although larger samples might have shown differences, results suggest that any such differences would have to be small and less important than differences in somatic symptoms and social functioning. Continuing physical discomfort up to six months after operation did not seem to induce appreciable emotional upset in our patients, despite suggestions that fatigue induced by radiotherapy might be a major source of distress. ${ }^{621}$

Nevertheless, several patients reported that radiotherapy was emotionally upsetting. Some were intimidated by the radiotherapy equipment, two stating that they could never go through the treatment again. A few were distressed by seeing patients with advanced cancer. One patient feared that radiotherapy might cause long term physical harm. Other researchers have reported similar findings. ${ }^{6}{ }^{2122} 23$ Two patients who had felt sick during radiotherapy experienced conditioned reflex nausea on re-entering the treatment centre. At six months, when the 23 patients who had received 
radiotherapy alone were asked their opinions about their treatment, five described it as having been "very severe," "very unpleasant," "very rough," "horrible," and "terrible." At least half of the 23 patients, however, had no particular complaints about their treatment. Reassurance by the radiographers seemed to allay the fears of some of them. One patient said that she felt insecure after completing her treatment because she was no longer getting frequent check ups. On the whole psychological upset associated with radiotherapy seemed to be short lived and did not usually result in increased ratings of anxiety or depression at three months.

Although most patients who did not have radiotherapy interpreted their lack of postoperative treatment favourably, there were notable exceptions. A few became anxious because they had not received further treatment, but unlike those receiving radiotherapy they attended clinics infrequently after operation so that there were fewer opportunities to seek reassurance or support. For example, one patient feared that her illness was too serious to justify any further intervention. She began to experience panic attacks, which she interpreted as a sign of metastatic disease; several weeks elapsed before she was reassured. Interestingly, Fallowfield et al recently reported that certain patients who had had breast conservation instead of mastectomy became anxious because they feared that treatment had been inadequate, and they emphasised the need for counselling in patients treated conservatively. ${ }^{24}$

This study confirms that a substantial minority of patients experience psychological morbidity after mastectomy, though perhaps of less degree than that reported in previous studies. ${ }^{3}$ Postoperative radiotherapy is sometimes very unpleasant and is associated with an increased incidence of physical symptoms and impaired social functioning. Compared with no postoperative treatment, however, radiotherapy does not seem to induce an excess of depression or anxiety. The psychological morbidity induced by a three week course of radiotherapy after mastectomy is of short duration and is probably overshadowed by the emotional distress associated with fear of cancer and its consequences.

This study was supported by grants from the Cancer Research Campaign. We thank the patients for their cooperation, and members of the division of surgery, Victoria Infirmary, Glasgow, and Drs Agnes Russell and Tim Habeshaw of the Institute of Radiotherapeutics and Oncology, Western Infirmary, Glasgow, for permission to interview patients under their care.
Dr Peter Maguire, University of Manchester, kindly allowed us to use his rating scales.

\section{References}

1 Anonymous. Postoperative radiotherapy in breast cancer [Editorial]. Br Med f 1981;282:1498-9. 2 Gazet J-C, Rainsbury RM, Ford FT, Powles TJ. Survey of treatment of primary breast cancer in Great Britain. Br Med f 1985;289:1793-5.

3 Morris T. Psychological adjustment to mastectomy. Cancer Treat Rev 1979;6:41-61.

4 Hughson AVM, Cooper AF, McArdle CS, Smith DC. Psychological impact of adjuvant chemotherapy in the first two years after mastectomy. Br Med $\mathcal{F} 1986 ; 292: 1268-71$.

5 Maguire GP, Tait A, Brooke M, Thomas C, Howat JMT, Sellwood R. Psychiatric morbidity and physical toxicity associated with adjuvant chemotherapy after mastectomy. $\mathrm{Br}$ Med $\mathrm{J}$ 1980;281:1179-80.

6 Holland J, Rowland J, Lebovits A, Rusalem R. Reactions to cancer treatment: assessment of the emotional response to adjuvant radiotherapy as a guide to planned intervention. Psychiatr Clin North Am 1979;2:347-57.

7 Maguire P. The psychological and social sequelae of mastectomy. In: Howells JG, ed. Modern perspectives in the psychiatric aspects of surgery. New York: Bruner Maisels, 1976:390-421.

8 Silberfarb PM, Maurer LH, Crouthamel C. Psychosocial aspects of neoplastic disease. I. Functional status of breast cancer patients during different treatment regimens. Am f Psychiatry Functional status

9 Gyllensköld K. (Transl Crampton P.) Breast cancer-the psychological effects of the disease and its treatment. London and New York: Tavistock Publications, 1982:145-212.

10 Hughes J. Emotional reactions to the diagnosis and treatment of early breast cancer. $\mathcal{F}$ Psychosom Res 1982;26:277-83.

11 Maguire GP, Lee EG, Bevington DJ, Küchemann C, Crabtree RJ, Cornell C. Psychiatric problems in the first year after mastectomy. Br Med f 1978;i:963-5.

12 Snaith RP, Bridge GW, Hamilton $M$. The Leeds scales for the self assessment of anxiety and depression. Br f Psychiatry 1976;128:156-65.

13 Goldberg DP. Manual of the general health questionnaire. Windsor: NFER Publishing, 1979.

14 Eysenck HJ, Eysenck SGB. Manual of the Eysenck personality inventory. London: University of London Press, 1964.

15 Raven JC. Guide to using the Mill Hill vocabulary scale with the progressive matrices scale. London: Lewis, 1958.

16 Peck DF. The conversion of progressive matrices and Mill Hill vocabulary raw scores into deviation IQ's. $\mathcal{F}$ Clin Psychol 1970;26:67-70.

17 McArdle CS, Crawford D, Dykes EH, et al. Adjuvant radiotherapy and chemotherapy in breast cancer. Br $\mathcal{J}$ Surg 1986;73:264-6.

8 Cohen J. Weighted kappa: nominal scale agreement with provision for scaled disagreement or partial credit. Psychol Bull 1968;70:213-20.

9 Armitage P. Statistical methods in medical research. Oxford: Blackwell Scientific Publications, 1971: 129-31.

20 Morris T, Greer HS, White P. Psychological and social adjustment to mastectomy: a two-year follow-up study. Cancer 1977;40:2381-7.

21 Greer S. Cancer: psychiatric aspects. In: Granville-Grossman K, ed. Recent advances in clinical psychiatry. Edinburgh: Churchill Livingstone, 1985:87-104.

22 Peck A, Boland J. Emotional reactions to radiation treatment. Cancer 1977;40:180-4.

23 Forester BM, Kornfeld DS, Fleiss J. Psychiatric aspects of radiotherapy. Am 7 Psychiatry 1978;135:960-3.

24 Fallowfield LJ, Baum M, Maguire GP. Effects of breast conservation on psychological morbidity associated with diagnosis and treatment of early breast cancer. Br Med F 1986;293:1331-4.

(Accepted 2 April 1987)

\title{
Ovarian neoplasms, functional ovarian cysts, and oral contraceptives
}

\author{
MARTIN VESSEY, ALISON METCALFE, CLIVE WELLS, KLIM MCPHERSON, \\ CAROLYN WESTHOFF, DAVID YEATES
}

\begin{abstract}
The incidence of ovarian neoplasms and functional ovarian cysts diagnosed at laparotomy or laparoscopy among the 17000 women taking part in the Oxford Family Planning Association contraceptive study was investigated. Epithelial cancer of the ovary was only $25 \%$ as common among those who had ever taken oral contraceptives as those who had never done so (95\% confidence interval $8 \%$ to $67 \%$ ). There was little evidence of any important association between use of oral contraceptives and benign teratoma or cystadenoma. Functional cysts of the ovary occurred much less commonly in women who had recently (in the six months preceding diagnosis) taken combined oral contraceptives (but not in those who had taken progestogen only oral contraceptives) than in those who had never taken oral con-
\end{abstract}

traceptives or had taken them in the past. This protective effect was more pronounced for corpus luteum cysts $(78 \%$ reduction; $95 \%$ confidence interval $47 \%$ to $93 \%$ ) than for follicular cysts (49\% reduction; $95 \%$ confidence interval $20 \%$ to $70 \%$ ).

It is estimated that about 28 (95\% confidence interval 16 to 35$)$ operations for functional ovarian cysts are avoided among every 100000 women who take oral contraceptives each year.

\section{Introduction}

The protective effect of combined oral contraceptives against epithelial cancer of the ovary has been described in a series of casecontrol studies. ${ }^{1}$ There is also some evidence that these preparations 